\title{
Crown Lengthening Procedure: Various Techniques A Case Series
}

\author{
${ }^{1}$ Saihas B. Pawar, ${ }^{2}$ P.S.Rakhewar, ${ }^{3}$ Lisa Chacko, ${ }^{4}$ Sneha S. Walkar
}

\begin{abstract}
Introduction: Crown lengthening is the process by which the extent of supragingival tooth structure is increased. It is used to for aesthetic of functional (retention) purposes.

Method: Various techniques are used for crown lengthening purpose. It is important to choose which technique should be employed for that patient. Techniques such as gingivectomy, flap with osseous reduction, apically positioned flap with or without osseous reduction have been used for crown lengthening procedure.

Case Report: This article consists, series of 4 case reports highlighting its need according to the requirement and demand of the situation.

Discussion: Crown Lengthening is a surgical procedure that requires exposure of adequate tooth structure for restorative procedures. The cases discussed here have been treated with various techniques and methods. All cases discussed here were treated in such a way so as to avoid any violation to Biologic Width that can have deleterious effect on periodontium leading to gingival inflammation, loss of attachment and alveolar resorption.

Keywords: crown lengthening, biological width, gingivectomy, apically positioned flap
\end{abstract}

\section{Introduction}

Crown lengthening: A surgical procedure designed to increase the extent of supragingival tooth structure for restorative or esthetic purposes by apically positioning the gingival margin, removing supporting bone, or both and may be accomplished by orthodontic tooth movement. ${ }^{(1)}$ The concept of tooth lengthening was first introduced by D. W. Cohen (1962) ${ }^{(2)}$. The procedure is based on two principles: BIOLOGIC WIDTH (BW) establishment and maintenance of adequate KERATINIZED GINGIVA (KG) around the tooth.

Biological width (BW) : The BW is defined as the dimension of soft tissue that is attached to the portion of the tooth coronal to the alveolar bone crest. ${ }^{(3,4,5)}$. It was first described by Sicher 1959 and later by Gargiulo and collegues in 1961.Vacek and colleagues $(1994)^{(6)}$ suggested that - BW increases anterioposteriorly $(1.75 \mathrm{~mm}-2.08 \mathrm{~mm})$.Kois 1994 and Spear 1999 suggested that the Interproximally BW is around $(4.5 \mathrm{~mm}-5.5 \mathrm{~mm})$

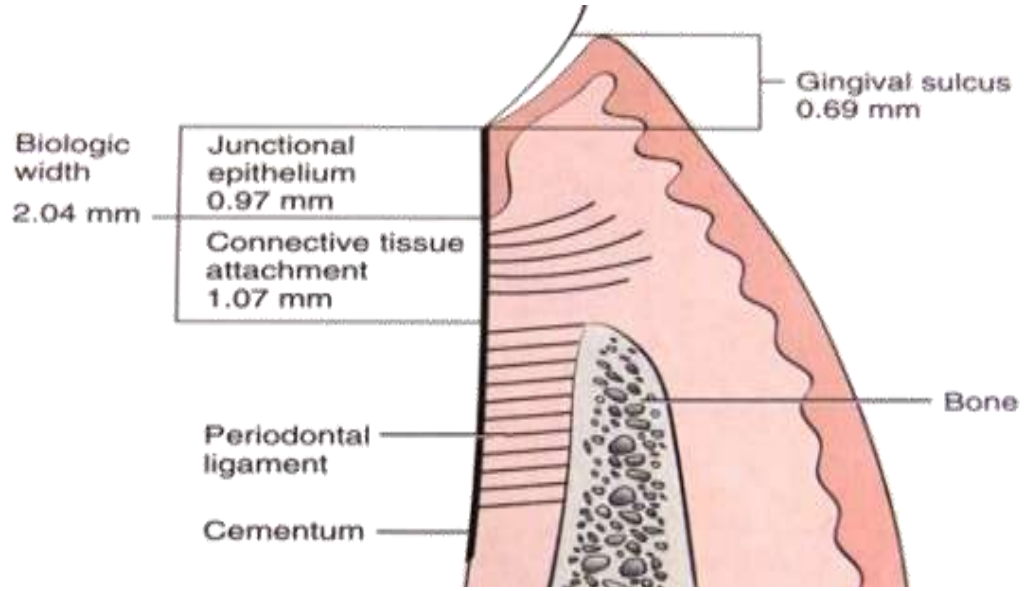

Indications : ${ }^{(7)}$

- Restorative needs

- To increase clinical crown height lost due to caries, fracture or wear

- To access Subgingival caries

- To produce a 'ferrule' for post crown provision

- To access a perforation in the coronalthird of the root

- To relocate margin of restorations that are impinging on biological width.

- Aesthetics 
- Short teeth

- Uneven gingival contour

- Gummy smile.

Contra-Indications \& Limiting Factors : ${ }^{(8)}$

- Inadequate crown to root ratio

- Non restorability of caries or root fracture

- Esthetic compromise

- High furcation

- Inadequate predictability

- Tooth arch relationship inadequacy

- Compromising of adjacent periodontium or esthetics

- Insufficient restorative space

- No maintainability

\section{Classification - esthetic \& functional}
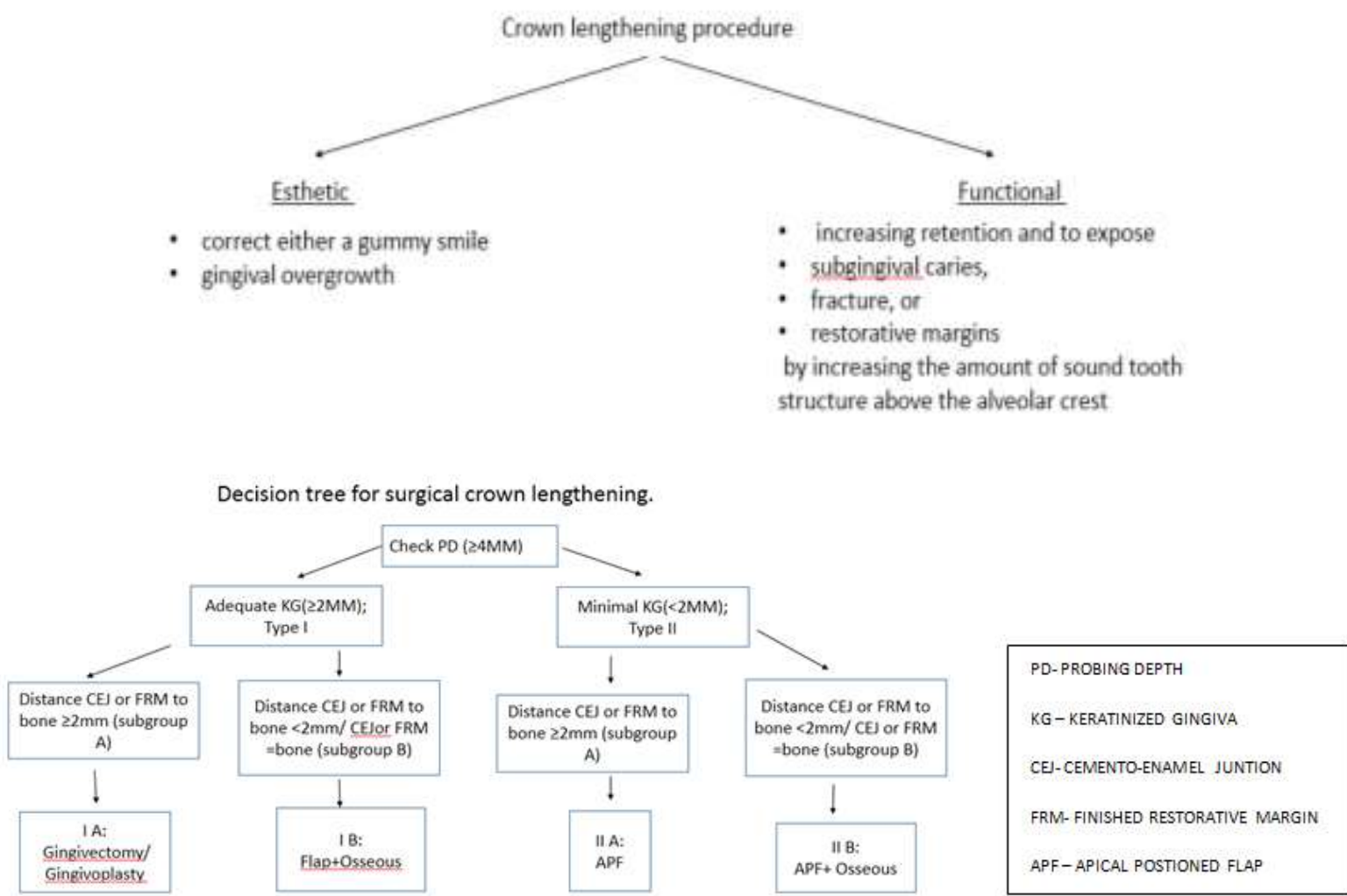

Decision Tree For Surgical Crown Lengthening : ${ }^{(9)}$

Based on the width of keratinized gingiva (adequate / inadequate), Marianne Ong et al has proposed a decision tree. This decision tree helps the clinician to choose which mode of procedure of crown lengthening is to be followed. 
Aim:

The aim of this case series is to highlight various principles of crown lengthening by presenting 4 case reports.

Initial treatment phase:

\section{Method}

- Oral prophylaxis and oral hygiene instructions along with the caries control are mandatory in the initial treatment phase.

- If endodontic therapy is indicated, it should be completed.

- Initial tooth preparation should be completed if possible and a provisional restoration placed to determine the amount of crown lengthening required.

General principles in crown lengthening procedure.

- Regardless of the surgical technique chosen, the final result should provide an adequate amount of tooth exposure to meet the restorative requirements.

- The actual amount will vary depending on the demands placed on the tooth.

- A tooth that will serve as an abutment for a fixed partial denture or a removable partial denture generally will require more tooth length for greater retention than receiving a simple restoration

- The minimal amount of tooth length needed supracrestally is $3-5 \mathrm{~mm}$.

- The surgical crown lengthening procedure depends mainly on the band of attached gingiva and the thickness of marginal alveolar bone.

- It is important to maintain an adequate dimension of gingiva around the tooth being surgically lengthened

- If there is a sufficient band of attached gingiva and thick gingival tissue on the marginal bone then only the soft tissue is removed.

- However if the band of the attached gingiva is insufficient then apically positioned flap surgery is used.

- Ideally there should be $5 \mathrm{~mm}$ of gingiva ( $3 \mathrm{~mm}$ attached and $2 \mathrm{~mm}$ free) in an apicocoronal dimension when restoration margins will be placed subgingivally.

- If less than this amount is present, there is risk for future gingival recession and restoration margin exposure. In cases with minimal gingiva, care should be taken to retain the entire band of marginal gingiva.

\section{Various Techniques For Performing The Crown Lengthening Surgery : ${ }^{(10,11)}$ \\ Surgical \\ - $\quad$ Gingivectomy- Conventional (Surgical), Laser, Electro cautery. \\ - $\quad$ Flap with osseous reduction \\ - Apical positioning of flap with or without ostectomy}

\section{Non-surgical}

- $\quad$ Orthodontic Treatment Combined

\section{Case 1: Gingivectomy:}

A 23 year old Patient came to the Department of Periodontology and Implantology, SMBT Dental College, Sangamner, Maharashtra with a chief complain of excessive gingival display, with a Probing Depth(PD) of about $5 \mathrm{~mm}$ and with an adequate width of keratinized gingiva, Distance CEJ to bone $\geq 2 \mathrm{~mm}$. Hence a gingivectomy procedure was preferred. This case was classified under esthetic ${ }^{(12,13,14,)}$ crown lengthening procedure.
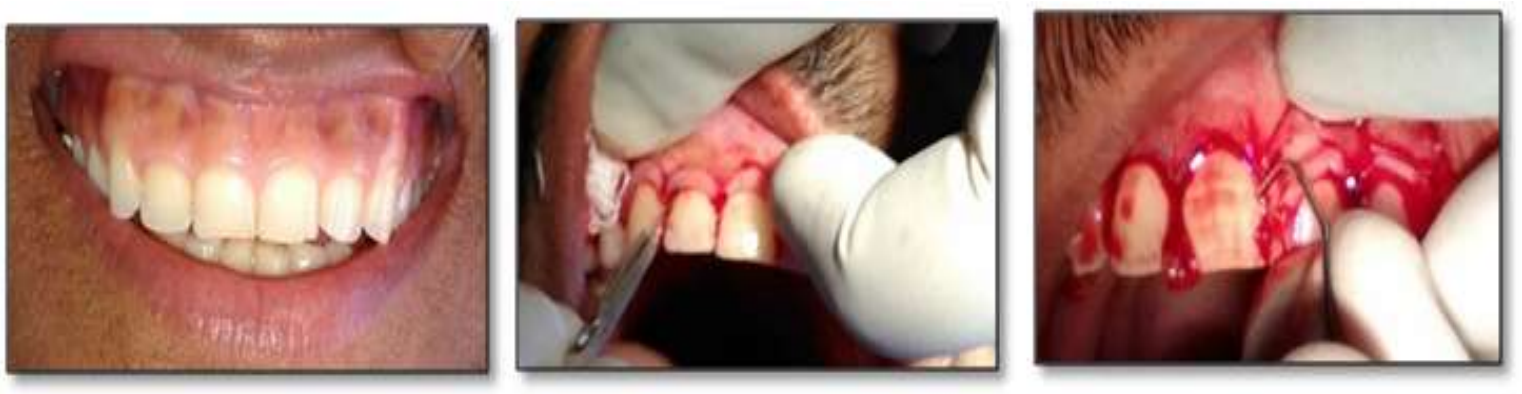

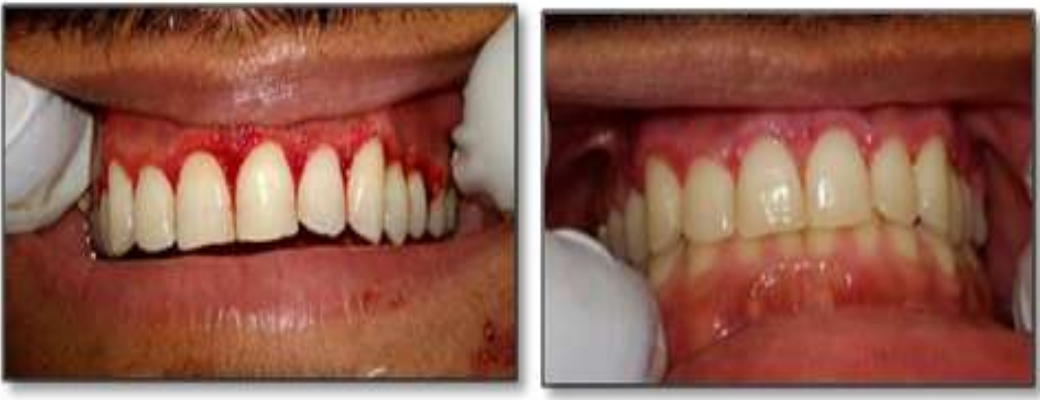

a.Preoperative, b. incisions f. 1 month follow-up

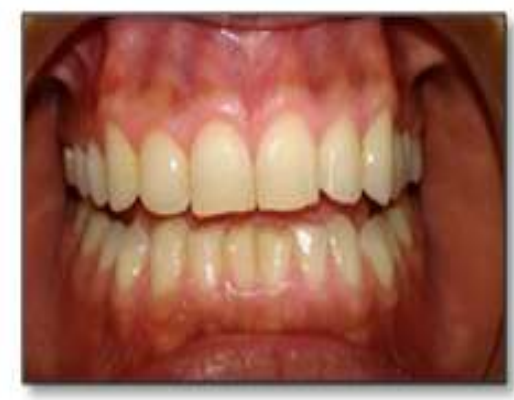

e. 10 days follow-up,

\section{Technique:}

On clinical evaluation the patient showed a high upper lip line. Careful evaluation of the location and thickness of the underlying bone was completed prior to beginning this procedure. The periodontal probe was used to perform bone sounding after administration of local anesthesia to rule out the necessity of osseous surgery. Pockets were marked using a pocket marker.Bard-parker blades no. 11 and 12, was used for the incisions on the facial surfaces.The incision was started apical to the gingival margin and was directed coronally. The incision was as close as possible to the bone without exposing it, to remove the soft tissue coronal to the bone.The incision was beveled at approximately 45 degrees to the tooth surface and should recreate as far as possible the normal pattern of the gingiva. The excess gingiva was removed and cleaned, and a post-surgical pack was placed.Patient was recalled after 10days for removal of post-surgical pack, and then after 1 month for follow-up. The increase in the extent of supragingival tooth structure was about $3 \mathrm{~mm}$ throughout after 1 month.

\section{Case 2: Flap With Osseous Reduction : ${ }^{(15)}$}

A 62 year old patient was referred for crown lengthening procedure to the Department of Periodontology and implantology for crown lengthening procedure, SMBT Dental College, Sangamner, Maharashtra, on examination PD was $4 \mathrm{~mm}$, the width of keratinized gingiva was Adequate $\mathrm{KG}(\geq 2 \mathrm{MM})$; but the distance from FRM to bone $<2 \mathrm{~mm}$. Hence flap procedure with osseous reduction was preferred. This case was classified under functional crown lengthening procedure.

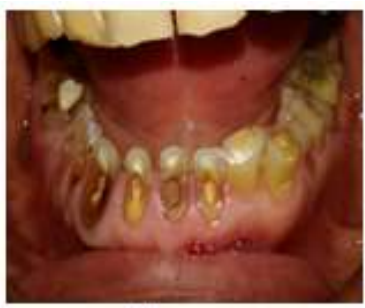

A

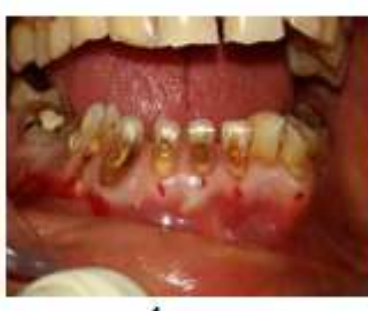

b

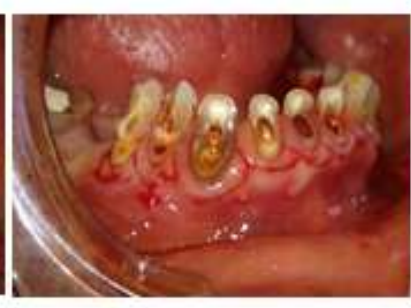

C

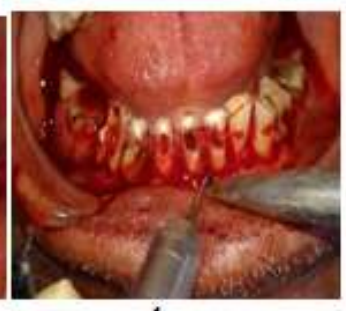

d

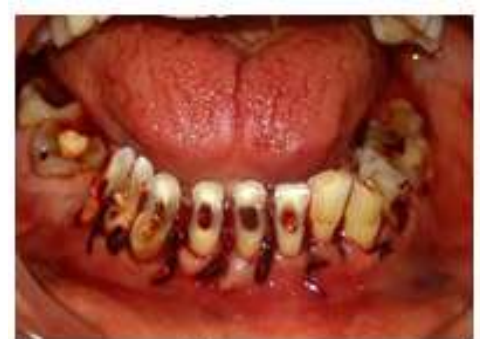

E

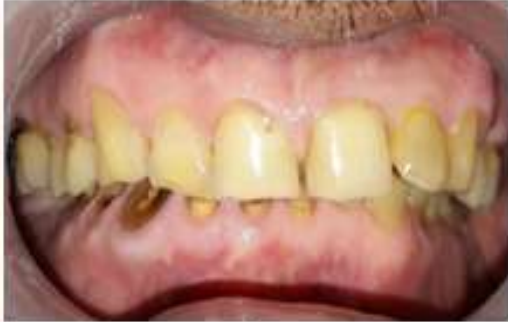

f

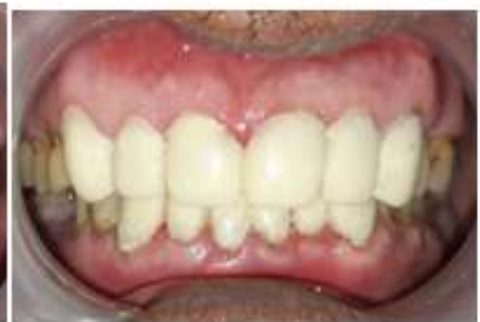

G

a. preoperative b. bleeding points c. incisions d. flap refection with bone reduction e. suture placement f. 1 month postoperative 


\section{Technique:}

The patient was carefully evaluated. Local anesthesia was administered and bone sounding was carried out.As the distance from the finished restorative margin to the alveolar bone was less than $2 \mathrm{~mm}$, hence there was need for osseous reduction.Internal bevel incision was given and a full thickness flap was reflected.The underlying bone was reduced using a diamond bur with ample of irrigation with saline so as to achieve a proper dimension of biological width and to expose the required tooth length in a scalloped fashion to follow the desired contour of the overlying gingiva.The gingiva was recontoured using a scissor and the flap was sutured back. The patient was recalled after 10days for suture removal.The increase in the extent of supragingival tooth structure was about $1-2 \mathrm{~mm}$ throughout after 1 month.

\section{Case 3: apical positioned flap without osseous reduction:}

A 32 year old male patient reported to the Department of Periodontology and Implantology, SMBT Dental College, Sangamner, Maharashtra, for crown lengthening procedure in upper right back region of the jaw. On clinical examination with $13,14 \& 15$, the width of keratinized gingiva was inadequate, $\mathrm{KG}(<2 \mathrm{MM})$; and the distance from or FRM to bone $\geq 2 \mathrm{~mm}$, hence an apically displaced flap without osseous reduction was preferred. This case was classified under functional crown lengthening procedure.

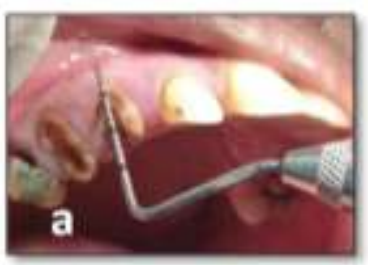

a. preoperative

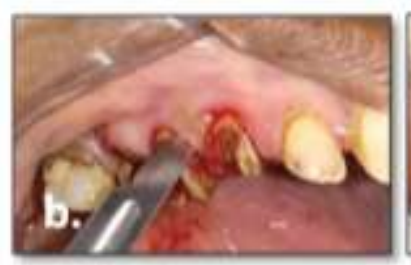

b. incision

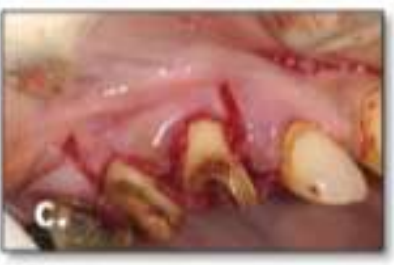

c. apically positioned flap

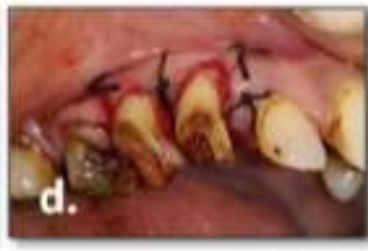

d. suture placement

\section{Technique:}

The patient was carefully evaluated. Local anesthesia was administered and bone sounding was carried out. crevicular incision was given, followed by the initial elevation of the flap.As the distance from the finished restorative margin to the alveolar bone was more than $2 \mathrm{~mm}$ there was no need for osseous reduction. Vertical incisions were placed which extends beyond the mucogingival junction and a full thickness flap was raised.The flap was displaced apical to its original position. The gingiva was recontoured using a pair of scissors. Periosteal sutures were placed to prevent the further apical displacement of the flap and periodontal pack was placed.Patient was recalled after 10days for removal of sutures and periodontal pack. The increase in the extent of supragingival tooth structure was about $2 \mathrm{~mm}$.

\section{Case 4: Apical Positioned Flap With Osseous Reduction : ${ }^{(16)}$}

A 34 year old female patient reported to the Department of Conservative Dentistry \&Endodontics, SMBT Dental College, Sangamner, Maharashtra, with the chief complaint of carious teeth in upper left back region of the jaw. Clinical examination revealed cariously fractured tooth extending subgingivally with 23,24 . The patient was checked and referred to Department of Periodontology and implantology for crown lengthening procedure, on examination the width of keratinized gingiva was inadequate, $\mathrm{KG}(<2 \mathrm{MM})$, and the distance from FRM to bone $<2 \mathrm{~mm}$, hence a apical displaced flap with osseous reduction was preferred. This case was classified under functional crown lengthening procedure.
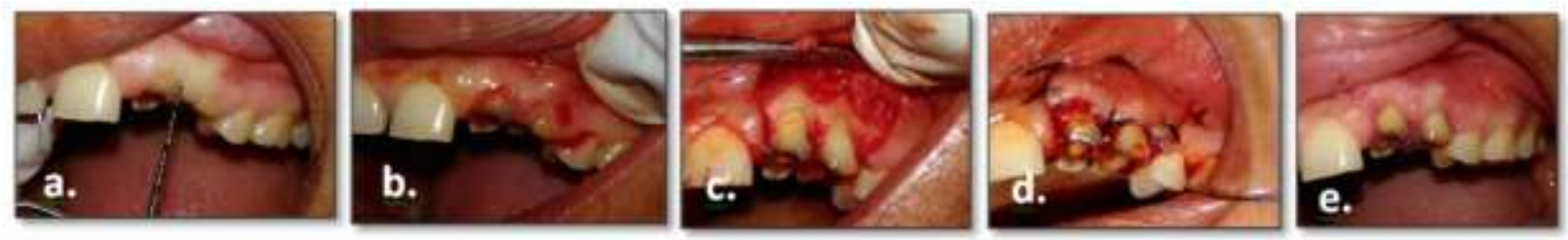

a. preoperative b. incision c. apically positioned flap d. suture placement e. postoperative 1 month.

\section{Technique:}

An internal bevel incision was made to preserve as much of the keratinized and attached gingiva as possible, the incision should be no more than about $1 \mathrm{~mm}$ from the crest of the gingiva and directed towards the crest of the alveolar bone. Crevicular incisions were made, followed by the initial elevation of the flap and then 
the interdental incision are placed and the wedge of tissue formed was removed.Vertical incisions were placed which extends beyond the mucogingival junction. An apical positioned flap was raised. The underlying bone was reduced using a diamond bur with ample of irrigation with saline so as to achieve a proper dimension of biological width and to expose the required tooth length in a scalloped fashion to follow the desired contour of the overlying gingiva.The flap was displaced apical to its original position. The gingiva was recontoured using a pair of scissors. Periosteal sutures were placed to prevent the further apical displacement of the flap and periodontal pack was placed. Patient was recalled after 10days for removal of sutures and periodontal pack and for follow-up after 1 month..The increase in the extent of supragingival tooth structure was about $3 \mathrm{~mm}$.

\section{Discussion}

Crown Lengthening is a surgical procedure that requires exposure of adequate tooth structure for restorative procedures. The dentogingival junction has been described as a functional unit composed of the connective tissue attachment of the gingiva and the epithelial attachment.Gargiuio et al reported that the connective tissue attachment varied in length from, 0 to $6.84 \mathrm{~mm}$ with a mean of $1.07 \mathrm{~mm}$; this measurement combined with the mean length of the epithelial attachment $(0.97 \mathrm{~mm})$ has been called the physiologic dentogingivai junction, or biologic width $(2.04)^{(6)}$. The physiologic location of the biologic width can vary with age, tooth migration due to loss of arch or occlusal integrity, or orthodontic treatment.

Violation of the biologic width is a common occurrence in the practice of restorative dentistry, e.g. placement of a deep subgingival restoration. The cases discussed here have been treated with various techniques and methods. All cases discussed here were treated in such a way so as to avoid any violation to Biologic Width that can have deleterious effect on periodontium leading to gingival inflammation, loss of attachment and alveolar resorption. The case 1 is treated as per the decision tree, that is by gingivectomy procedure since the width of keratinized gingiva was adequate and the distance from the CEJ/ Finished restorative margin (FRM) was more than $2 \mathrm{~mm}$. The case 2 was treated with flap with osseous reduction as the width of keratinized gingiva was adequate but the distance from FRM to the alveolar bone was $\leq 2 \mathrm{~mm}$. The case 3 was treated with apically positioning the flap and without osseous reduction as the width of keratinized gingiva was inadequate, but the distance from the FRM to the alveolar bone was $\geq 2 \mathrm{~mm}$. The case 4 required apical positioning of the flap along with osseous reduction as the width of keratinized gingiva was inadequate as well as the distance from the FRM to the alveolar bone was $\leq 2 \mathrm{~mm}$.

\section{Conclusion}

This article reviews esthetic and restorative (or functional) crown-lengthening procedures and highlights their indications and differences. The decision trees are presented to aid the clinician in the decisionmaking process of when these procedures should be used.Clinical crown lengthening is useful procedure to provide tooth length for proper restoration of a tooth without compromising the periodontium or the retentive qualities of the restoration. It is also useful for enhancing maxillary anterior esthetics.Crown lengthening may be as simple as a limited removal of soft tissue or as complex as orthodontic extrusion followed by flap with osseous surgery on a tooth requiring endodontic therapy.Careful evaluation, case selection, treatment planning and surgical treatment achieve results that meet the functional and esthetic challenges of current dental practice.Improper management of the periodontal tissues during restorative procedures is common, but often overlooked, cause of failure. When a restoration is placed, the preservation of an intact, healthy periodontium is necessary to maintain the tooth or teeth being restored.Predictable long-term restorative success requires a combination of restorative principles with the correct management of the periodontal tissues.

[1]. GlossaryOfPeriodontalTerms2001Edition.

\section{Reference}

[2]. Cohen DW. Lecture, Walter Reed Medical Center 1962 June 3

[3]. Gargiulo AW, Wentz FM, Orban B. Dimensions and relations of the dentogingival junction in humans. J Periodontol. 1961; 32:261-

[4]. Gargiulo AW, Wentz FM, Orban B. Dimensions and relations of the dentogingival junction in humans. J Periodontol 1961; 32:261267.

[5]. Vacek JS, Gher ME, Assad DA, Richardson AC, Giambarresi LI. The dimensions of the human dentogingival junction. Int J Periodontics Restorative Dent 1994; 14:154-165.

[6]. The Dimensions of the Human Dentogingivai Junction, Jomes S. Vacek, The International Journal of Periodontics \& Restorative Dentistry, The International Journal of Periodontics \& Restorative Dentistry

[7]. Camargo PM, Melnick PR, Camargo LM. Clinical Crown Lengthening in Esthetic Zone. C D A Journal. 2007; $35(7): 487-498$

[8]. Cohen ES. Crown lengthening. Atlas of Cosmetic \& reconstructive periodontal surgery. Third edition

[9]. Crown Lengthening Revisited, Marianne Ong, Shih-Chang Tseng, and Hom-Lay Wang, Clinical Advances in Periodontics, Vol. 1, No. 3, November 2011

[10]. Wennstrom J L, PiniPrato GP. Mucogingival therapy-periodontal plastic surgery; Clinical Periodontlogy\& Implant dentistry.4th edition

[11]. HH Takei. Preparation of the periodontium for restorative dentistry. Clinical Periodontology. Tenth Edition. 
[12]. Aesthetic crown lengthening: classification, biologic rationale, and treatment planning considerations, Ernesto A. Lee, DMD, Dr Cir Dent, PractProcedAesthet Dent 2004;16(10):769-778

[13]. Aesthetic Surgical Crown Lengthening Procedure, Pablo Santos de Oliveira Hindawi Publishing Corporation,Case Reports in Dentistry, Volume 2015, Article ID437412, 4 pages

[14]. Surgical crown lengthening procedure to enhance esthetics, The Internotional Journal of Periodontics \& Restorative Dentistry, Volume 19, Number 4.1999

[15]. Crown lengthening: A 6 month study, J. Periodontol, September 2004, vol 75, number 9. Deas, Moritz, McDonnell, Powell, Mealey.

[16]. Crown Lengthening Surgery (Cls): A Mini Review \& Series of 4 Case Reports, Indian Journal of Dental Sciences. December 2014 Issue: 5, Vol.:6 